PS $\quad$ PROCEEDINGS

\title{
Measurement of high energy cosmic rays by the new Tibet hybrid experiment
}

M. Amenomori, ${ }^{a}$ X. J. Bi, ${ }^{b}$ D. Chen, ${ }^{c}$ T. L. Chen, ${ }^{d}$ W. Y. Chen, ${ }^{b}$ S. W. Cui, ${ }^{e}$ Danzengluobu, ${ }^{d}$ L. K. Ding, ${ }^{b}$ C. F. Feng, ${ }^{f}$ Zhaoyang Feng, ${ }^{b}$ Z. Y. Feng, ${ }^{g}$ Q. B. Gou, ${ }^{b}$ Y. Q. Guo,${ }^{b}$ H. H. He, ${ }^{b}$ Z. T. He, ${ }^{e}$ K. Hibino, ${ }^{h}$ N. Hotta, ${ }^{i}$ Haibing Hu, ${ }^{d}$ H. B. Hu, ${ }^{b}$ J. Huang ${ }^{*}{ }^{b}$ H. Y. Jia, ${ }^{g}$ L. Jiang, ${ }^{b}$ F. Kajino, ${ }^{j}$ K. Kasahara, ${ }^{k}$ Y. Katayose, ${ }^{l}$ C. Kato, ${ }^{m}$ K. Kawata, ${ }^{n}$ M. Kozai, ${ }^{o m}$ Labaciren, ${ }^{d}$ G. M. Le, ${ }^{p}$ A. F. Li, ${ }^{q f b}$ H. J. Li, ${ }^{d}$ W. J. Li, ${ }^{b g}$ C. Liu, ${ }^{b}$ J. S. Liu, ${ }^{b}$ M. Y. Liu, ${ }^{d}$ H. Lu, ${ }^{b}$ X. R. Meng, ${ }^{d}$ T. Miyazaki, ${ }^{m}$ K. Mizutani, ${ }^{k r}$ K. Munakata, ${ }^{m}$ T. Nakajima, ${ }^{m}$ Y. Nakamura, ${ }^{m}$ H. Nanjo, ${ }^{a}$ M. Nishizawa, ${ }^{s}$ T. Niwa, ${ }^{m}$ M. Ohnishi, ${ }^{n}$ I. Ohta, ${ }^{t}$ S. Ozawa, ${ }^{k}$ X. L. Qian, ${ }^{f b}$ X. B. Qu, ${ }^{u}$ T. Saito, ${ }^{,}$T. Y. Saito, ${ }^{w}$ M. Sakata, ${ }^{j}$ T. K. Sako, ${ }^{x n}$ J. Shao, ${ }^{b f}$ M. Shibata, ${ }^{l}$ A. Shiomi, ${ }^{y}$ T. Shirai, ${ }^{h}$ H. Sugimoto, ${ }^{z}$ M. Takita, ${ }^{n}$ Y. H. Tan, ${ }^{b}$ N. Tateyama, ${ }^{h}$ S. Torii, ${ }^{k}$ H. Tsuchiya,${ }^{A}$ S. Udo, ${ }^{h}$ H. Wang,${ }^{b}$ H. R. Wu, ${ }^{b}$ L. Xue, ${ }^{f}$ Y. Yamamoto, ${ }^{j}$ K. Yamauchi, ${ }^{l}$ Z. Yang, ${ }^{b}$ A. F. Yuan, ${ }^{d}$ T. Yuda,${ }^{\dagger}$ L. M. Zhai, ${ }^{c}$ H. M. Zhang, ${ }^{b}$ J. L. Zhang, ${ }^{b}$ X. Y. Zhang, ${ }^{f}$ Y. Zhang, ${ }^{b}$ Yi Zhang, ${ }^{b}$ Ying Zhang, ${ }^{b}$ Zhaxisangzhu $^{d}$ and X. X. Zhou ${ }^{g}$ (The Tibet AS $\gamma$ Collaboration) 
${ }^{a}$ Department of Physics, Hirosaki University, Japan

${ }^{b}$ Key Laboratory of Particle Astrophysics, Institute of High Energy Physics, Chinese Academy of Sciences, China

${ }^{c}$ National Astronomical Observatories, Chinese Academy of Sciences, China

${ }^{d}$ Department of Mathematics and Physics, Tibet University, China

${ }^{e}$ Department of Physics, Hebei Normal University, China

${ }^{f}$ Department of Physics, Shandong University, China

${ }^{g}$ Institute of Modern Physics, SouthWest Jiaotong University, China

${ }^{h}$ Faculty of Engineering, Kanagawa University, Japan

${ }^{i}$ Faculty of Education, Utsunomiya University, Japan

${ }^{j}$ Department of Physics, Konan University, Japan

${ }^{k}$ Research Institute for Science and Engineering, Waseda University, Japan

${ }^{l}$ Faculty of Engineering, Yokohama National University, Japan

${ }^{m}$ Department of Physics, Shinshu University, Japan

${ }^{n}$ Institute for Cosmic Ray Research, The University of Tokyo, Japan

${ }^{\circ}$ Institute of Space and Astronautical Science, Japan Aerospace Exploration Agency (ISAS/JAXA),

Japan

${ }^{p}$ National Center for Space Weather, China Meteorological Administration, China

${ }^{q}$ School of Information Science and Engineering, Shandong Agriculture University, China

${ }^{r}$ Saitama University, Japan

${ }^{s}$ National Institute of Informatics, Japan

${ }^{t}$ Sakushin Gakuin University, Japan

${ }^{u}$ College of Science, China University of Petroleum, China

${ }^{v}$ Tokyo Metropolitan College of Industrial Technology, Japan

${ }^{w}$ Max-Planck-Institut für Physik, Deutschland

${ }^{x}$ Escuela de Ciencias Físicas y Nanotechnología, Yachay Tech, Ecuador

${ }^{y}$ College of Industrial Technology, Nihon University, Japan

${ }^{z}$ Shonan Institute of Technology, Japan

${ }^{A}$ Japan Atomic Energy Agency, Japan

E-mail: huangjing@ihep.ac.cn

We have started a new hybrid air shower experiment at Yangbajing (4300 m a.s.1.) in Tibet in February 2014. This new hybrid experiment consists of the YAC-II comprised of 124 core detectors placed in the form of a square grid of $1.9 \mathrm{~m}$ spacing covering about $500 \mathrm{~m}^{2}$, the Tibet-III air shower array with the total area of about $50,000 \mathrm{~m}^{2}$ and the underground MD array consisting of 80 cells, with the total area of about $4,200 \mathrm{~m}^{2}$. This hybrid-array system is used to observe air showers of high energy celestial gamma-ray origin and those of nuclear-component origin. In this paper, a short review of the experiment will be followed by an overview on the current results on energy spectrum and chemical composition of CRs and test of hadronic interaction models.

35th International Cosmic Ray Conference - ICRC2017

10-20 July, 2017

Bexco, Busan, Korea

\footnotetext{
* Speaker.

${ }^{\dagger}$ Deceased.
} 


\section{Introduction}

The all-particle energy spectrum of primary cosmic rays is well characterized by a power law $\mathrm{dN} / \mathrm{dE} \propto \mathrm{E}^{-\gamma}$ over many orders of magnitude, with $\gamma$ changes sharply from 2.7 to 3.1 at about 4 $\mathrm{PeV}$ [1]. Such structure of the all-particle energy spectrum is called the "knee", which is considered to be closely related to the origin, acceleration and propagation mechanism of cosmic rays. In order to explain the existence of the knee, many hypotheses and mechanisms have been proposed. Although all these approaches can well describe the knee structure, there are much discrepancies in the prediction of the individual components at the knee region [2], [3]. The difficulty in settling the discussions is due to a lack of the data on the energy spectra of individual CR components whose energies are too high to catch directly with detectors on board balloons or satellites because of extremely scarce flux. To challenge the measurement of chemical composition around the knee, we have developed a new air-shower core detector (YAC). One important improvement is to lower the detection threshold energy of primary particles to $\sim 10 \mathrm{TeV}$, about one order of magnitude smaller than the previous experiment [4]. With this improvement, the energy spectra of individual components measured by YAC will overlap with those of direct measurements [5], which may help us to examine the knee of light component, such as "proton knee" ;or "helium knee". Another important improvement of YAC is its ability to count the number of shower particles passing through each detector to a wide dynamic range of 1 to $10^{6}$ particles, making it possible to observe the primary cosmic rays in the energy range from $\sim 10 \mathrm{TeV}$ to $\sim 10 \mathrm{PeV}$. Its goals are to reconstruct the primary energy and composition of cosmic rays at the energies between $50 \mathrm{TeV}$ to $10^{16} \mathrm{eV}$ thereby allowing a detailed investigation of the expected "proton knee", "helium knee" and "iron knee" [6], [7]. In this paper, a short review of the experiment will be followed by an overview on the current results on the energy spectrum and chemical composition of CRs and test of hadronic interaction models.

\section{2. (Tibet-III + YAC-II + MD) experiment}

The new Tibet hybrid experiment (Tibet-III+YAC-II+MD) has been operated in Tibet, China, and data taking started from February 2014. This hybrid experiment currently consists of three types of detector array, including the Tibet AS array (Tibet-III), the Yangbajing AS core-detector array (YAC-II) and an underground water-Cherenkov muon-detector array (MD), as shown in Fig. 1.

The Tibet-III consists of 789 detectors, with a covering area $\sim 50,000 \mathrm{~m}^{2}$. The YAC-II consists of 124 detectors with a covering area of $\sim 500 \mathrm{~m}^{2}$. The $5 \mathrm{MD}$ pools consists of 80 cells, with a total area of $\sim 4,200 \mathrm{~m}^{2}$. YAC-II has inner 100 plastic scintillator units, arranged as an array of $10 \times 10$ grids ( $1.9 \mathrm{~m}$ spacing), each with an area of $50 \mathrm{~cm} \times 80 \mathrm{~cm}$, and outer 24 plastic scintillator units each with an area of $50 \mathrm{~cm} \times 100 \mathrm{~cm}$ arranged around the inner array. The outer 24 units are used to reject "non core" events whose shower cores are far from the YAC-II array. A lead plate of the thickness $3.5 \mathrm{~cm}$ is put on each YAC-II detector to convert high energy electrons and gammas in the cores into electromagnetic showers. Light signals from each scintillator are read out using two photomultiplier tubes of high gain (HAMAMATSU: R4125) and low gain (HAMAMATSU: R5325) to cover the wide dynamic range from 1 MIP (Minimum Ionization Particle) to $10^{6}$ MIPs. The YAC-II array has been constructed near the center of the Tibet-III, and operating simultaneously with the other arrays. For an air shower event, the Tibet-III provides the arrival direction $(\theta)$, and the air shower size $\left(N_{e}\right)$ which is interrelated to primary energy while the YAC-II mea- 
sures the high energy electromagnetic particles in the core region so as to obtain the characteristic parameters of air-shower cores. When a YAC event is triggered, its accompanying air shower is simultaneously recorded. The matching between YAC, AS and MD events is made by their arrival time stamps. The air-shower direction can be estimated with an error smaller than $0.2^{0}$ above 100 $\mathrm{TeV}$, and the primary energy resolution is estimated to be $12 \%$ at energies around $10^{15} \mathrm{eV}$ by our simulation.
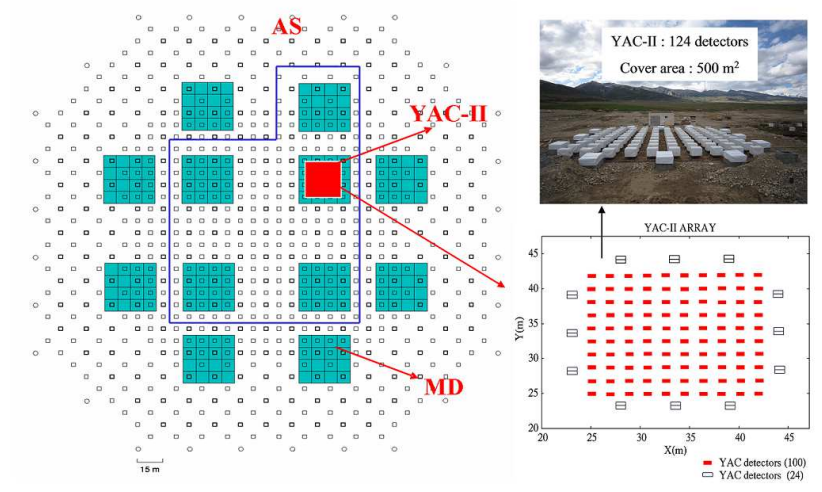

Figure 1: Schematic view of (Tibet-III+YAC-II+MD) array

\section{Monte Carlo Simulation}

A full Monte Carlo (MC) simulation has been carried out on the development of air showers in the atmosphere using the simulation code Corsika(version 7.3500) [9] including the interaction models EPOS-LHC, QGSJETII-04 and SIBYLL2.1. For the primary cosmic rays, we examined three primary composition models (shown in Fig.2), namely, "He-poor", "He-rich" and "Gaisserfit" models, in order to evaluate the systematic errors attributable to primary composition models $[6,7]$. The proton spectra of the former two models are fitted to the direct measurements at low energies and consistent with the spectrum obtained from the Tibet-EC experiment at higher energies. The He spectrum of He-poor model coincides with the results from RUNJOB, while the He spectrum of He-rich model coincides with the results from JACEE, ATIC2 and CREAM. The Gaisser-fit model fits to a higher He model (almost same as our He-rich model) at the low energy range and to the KASCADE-QGSJET data at high energy range in which light components ( $\mathrm{P}$ and $\mathrm{He}$ ) dominate in the chemical composition [8]. In this three composition models mentioned above, each component is summed up so as to match with the all-particle spectrum with a sharp knee, which was obtained with the Tibet-III air shower array [1]. The energy spectra of individual components (or mass groups) for three primary models are shown in Fig.2. It is seen that all the individual components of the three models in the low energy range (less than $100 \mathrm{TeV}$ ) are in good agreement with direct measurements while they differ significantly at higher energies. The all-particle spectra of three models, however, coincide with each other and reproduce the sharp knee [1] structure as well.

The detector responses to shower particles falling on the detectors of (YAC-II+Tibet-III+MD) array are calculated using the Geant4 [10], where the detector performance, trigger efficiency and effective area are adequately taken into account based on the experimental conditions. After reconstruction of each air shower event, we can obtain some characteristic parameters observed with the 


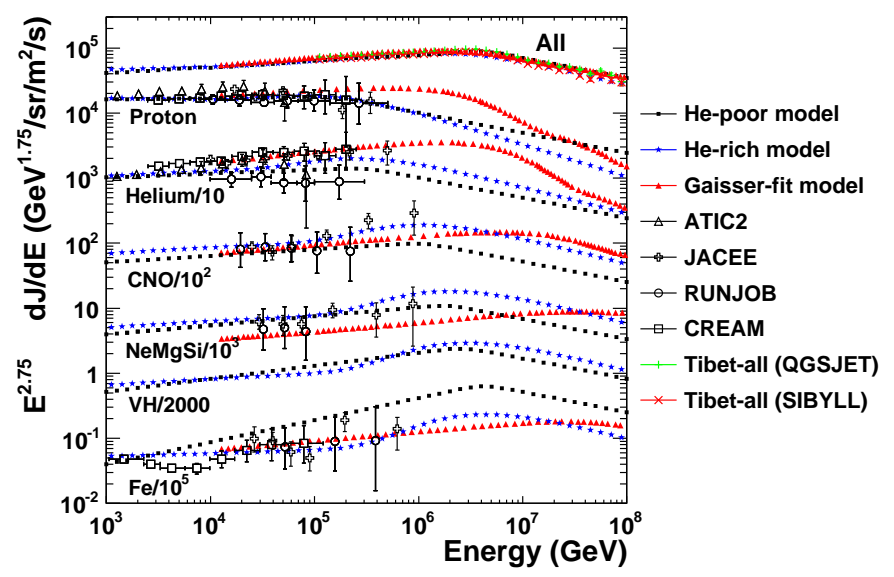

Figure 2: Primary cosmic-ray composition for He-poor, He-rich and Gaisser-fit models compared with those of direct measurements [7] and the sum of all components compared with the results obtained by the Tibet-III experiment [1] .

(YAC-II+Tibet-III+MD) array, and we find these parameters are very sensitive to different primary CR components.

\section{Analysis}

\subsection{Reconstruction of air-shower core events}

The reconstruction of air-shower core events mainly starts from the evaluation of the number of burst size $\left(N_{b}\right)$ under the lead plate of a YAC detector unit, that is the total energy loss in each scintillator divided by the single peak value, and then we obtain the following parameters reflecting the features of the core region:

$N_{h i t}$ - the number of "fired" detector units with $N_{b} \geq$ a given threshold value $\left(N_{b} \geq 100\right.$ in this paper);

$N_{b}{ }^{\text {top }}$ - the maximum burst size among the fired detectors;

$\sum N_{b}$ - the total burst size of all fired detector units;

$\langle R\rangle$ - the mean lateral spread, $\langle R\rangle=\sum r_{i} /\left(N_{h i t}-1\right)$;

$\left\langle N_{b} R\right\rangle$ - the mean energy-flow spread, $\left\langle N_{b} R\right\rangle=\sum\left(N_{b i} \times r_{i}\right) / N_{h i t}$, where $N_{b i}$ and $r_{i}$ are the burst size in the $i^{\text {th }}$ fired detector unit and the lateral distance from the burst center, which is calculated as $N_{b}$ weighted center of gravity, to the center of the $i^{\text {th }}$ fired detector, respectively.

We also tried to reconstruct other parameters reflecting the features of core region, and found that the five parameters mentioned above are basic and enough for the separation of primary cosmic-ray mass, though other parameters bring slight improvement to the separation of primary cosmic-ray mass.

\subsection{Reconstruction of air-shower events}

The same procedure as the experimental data analysis for air showers was applied to the MC data, i.e., the reconstruction of the core position, the arrival direction are described in [1]. In this work, the determination of the lateral distribution function of shower particles is very important, 
since the total number of effective particles in each event is estimated by fitting this function to the experimental data. In this work, we used the modified NKG function to determinate the lateral distribution. For details, see the paper [1]. Using the MC data obtained under the same conditions as the experiment, we found that this modified NKG function can be well fitted to the lateral distribution of shower particles obtained with the detectors with a lead plate of $5 \mathrm{~mm}$ thickness on its surface, and we found that the shower size resolutions estimated is about $5 \%$ around the primary energy of $1000 \mathrm{TeV}$. We are then able to obtain the correlation between the estimated shower size $N_{e}$ and the primary energy $E_{0}$ for each interaction models and composition models. We found there is less than $3 \%$ difference for the determination of the primary energy based on different hadronic interaction models [6].

\subsection{Reconstruction of Muon events}

Each cell of the MD array is composed of a concrete water tank of $7.2 \mathrm{~m}$ wide $\times 7.2 \mathrm{~m}$ long $\times 1.5 \mathrm{~m}$ deep, equipped with two downward-facing 20-inch-in-diameter PMTs (HAMAMATSU: R3600) on the ceiling. The reconstruction of the muon events was made by calculating the number of Cherenkov photons hitting on the PMTs, then we can get the number of muons at each MD pool as the total number of photons divided by the single peak value. Finally, we get the sum of muons $\left(N_{\mu}\right)$ "fired" in the MD array of an air-shower event.

\section{Results and Discussions}

\subsection{Sensitivity of ( YAC-II+Tibet-III + MD ) to measure the primary composition spectra}
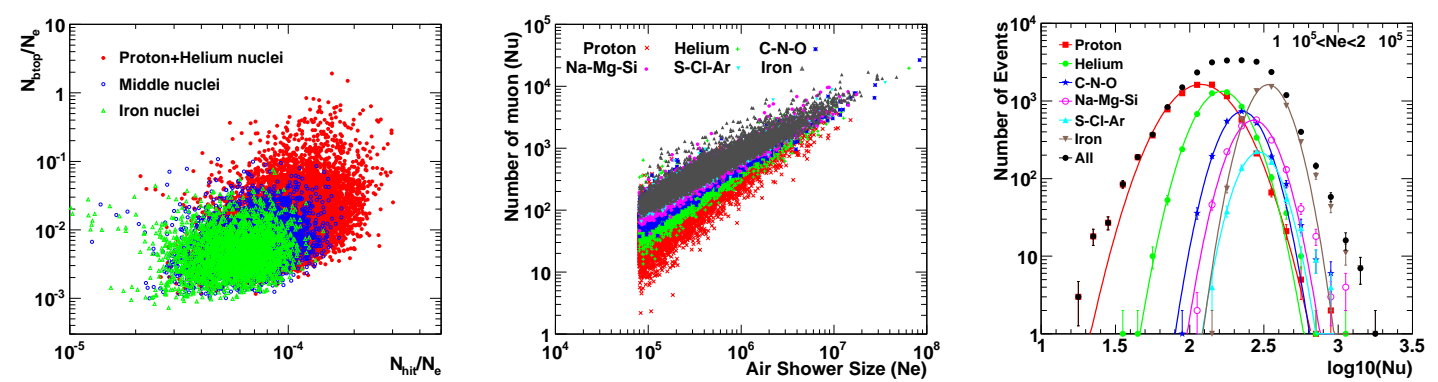

Figure 3: Left: The correlation between the $N_{\text {hit }}$ and $N_{b}^{\text {top }}$ parameters under QGSJET+He-poor model, both of which are normalized by $N_{e}$; Middle: The correlation between $N_{e}$ and $N_{\mu}$ observed with the ( YACII+Tibet-III + MD ) array; Right: $N_{\mu}$ distribution for various primary nuclei at $1 \times 10^{5}<N_{e}<2 \times 10^{5}$ bin.

Based on the above section 4.1, 4.2 and 4.3, we can obtain some parameters recorded by this new Tibet hybrid experiment (YAC-II+Tibet-III+MD) and it is found they are very sensitive to different primary cosmic-ray nuclei. An example of the sensitivity to the primary mass by the parameters from YAC-II array is shown in Fig.3(left figure), in which a correlation between $N_{h i t}$ and $N_{b}{ }^{\text {top }}$, both of them are normalized by $N_{e}$, is shown by different color for Proton+Helium, Middle and Iron nuclei under QGSJET+He-poor model. For the MD array, the sensitivity to the primary nuclei is seen in $N_{e}$ and $N_{\mu}$ correlation. Figure3 (middle figure) shows the correlation between $N_{e}$ and $N_{\mu}$ on each event observed with the (YAC-II+Tibet-III+MD) array based on QGSJET+He-poor 
model, and we can see that the distribution of $N_{\mu}$ and $N_{e}$ apparently depends on the primary nuclei. When we set $1 \times 10^{5}<N_{e}<2 \times 10^{5}$, the $N_{\mu}$ distribution strongly depends on primary mass as shown in the Fig.3 (right figure).

In this work, we use a feed-forward artificial neural network (ANN) method to separate Plike events, He-like events and Iron-like events by using the above reconstructed parameters. MC shows that this new hybrid experiment has a capability of measuring the primary energy spectrum for each of 4 mass groups of $\mathrm{P}, \mathrm{He}$, Iron and $4<\mathrm{A}<56$ at $50 \mathrm{TeV}-10 \mathrm{PeV}$ range covering the knee. For details, see the paper [6].

\subsection{Test of hadronic interaction models by (YAC-I+Tibet-III) hybrid experiment}

The interpretation of air showers is known to inevitably depends on the Monte Carlo simulations which are based on some hadronic interaction models and cosmic-ray composition models. At present, the simulation code CORSIKA that is comprehensively used in the ground-level cosmic-ray studies includes many interaction models. In this paper, we report our approach and the preliminary results to check the hadronic interaction models EPOS-LHC, QGSJETII-04 and SIBYLL2.1 at an energy region of $100 \mathrm{TeV}-1 \mathrm{PeV}$ using the data obtained by the prototype YACI (Yangbajing Air shower Core detector as the first stage). Data taking was made from May 2009 to February 2010, together with the Tibet-III array.

This prototype 16 YAC-I detectors were constructed near the center of the Tibet-III AS array with dense spacing. The response linearity of each YAC-I detector was calibrated by cosmic-ray single muons and by the accelerator beam (BEPC-LINAC) [11]. YAC-I is triggered when any one of 16 detectors records a local shower with a size of at least 20 MIPs. The event rate is about $30 \mathrm{~Hz}$. The total live time of our data set in present analysis is 169.65 days. Full MC simulation was made using three interaction models with primary energy above $5 \times 10^{13} \mathrm{eV}$. We examined three primary composition models namely, "He-poor", "He-rich" and "Gaisser-fit" models (shown in Fig.2 ). All detector responses is based on the detector simulation code Geant4 (version 9.5). The simulated events are passed through the same analysis chains as the experimental data. The statistics of high energy EAS core events are shown in Table1.

Table 1: Statistics of EAS core events in MC simulation and Experiment.

\begin{tabular}{ll}
\hline \hline Models & Core events \\
\hline SIBYLL2.1+He-rich & 64,331 \\
SIBYLL2.1+He-poor & 47,580 \\
QGSJETII-04+He-poor & 31,928 \\
EPOS-LHC+He-poor & 42,137 \\
EPOS-LHC+Gaisser-fit & 139,098 \\
EPOS-LHC+He-rich & 246,938 \\
\hline Expt.data & 5035 \\
\hline
\end{tabular}

YAC-I shows the ability and sensitivity in checking the hadronic interaction models in 100 $\mathrm{TeV}$ - $1 \mathrm{PeV}$ region. Following quantities of YAC-I are used to characterize an high energy AS core event (see sec.4.1): $N_{b}, \sum N_{b}, N_{b}^{t o p},\langle R\rangle$ and $\left\langle N_{b} R\right\rangle$. The comparison of some observable 

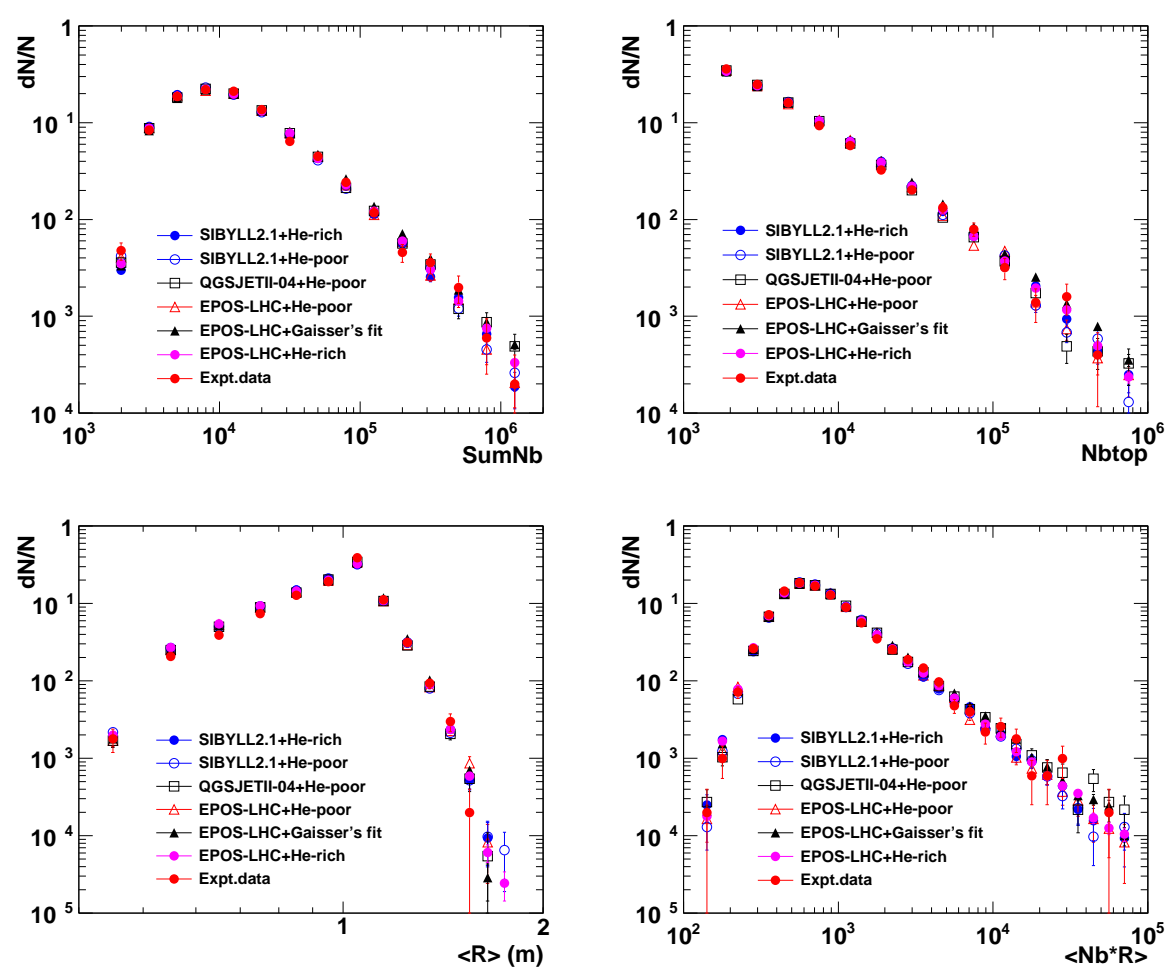

Figure 4: The comparison of some observable parameters $\left(\sum N_{b}, N_{b}{ }^{t o p},\langle R\rangle,\left\langle N_{b} R\right\rangle\right)$ between experimental data and MC simulation which are calculated for various combination of interaction and composition models.

parameters between YAC-I experimental data and MC simulation data which are calculated for various combination of interaction and primary composition models are given in Fig.4 and Fig.5.

Figure 4: This shows that the shape of each parameter distribution is almost same since these AS-core events are mainly a superposition of many EM cascade processes originated by gamma rays decayed from neutral pions. YAC-I experimental data are consistent with those MC simulation results, which means that there exist no detection biases within our experimental systematic uncertainty of about $10 \%$. It is to be noted that the shape shows a (cascade) scaling behavior, while its intensity depends on the primary composition model.

Figure 5: As seen in Fig.(a)-(b), there exist some discrepancies in the absolute intensities: 1) first, note that the three interaction models give almost same intensity when the primary is fixed to the "He-poor" model. This results suggest that the difference among three interaction models are very small, say at most $10 \%$ since almost all high energy cores are produced by the same primaryproton spectrum (He contribution is estimated to be very small [6]). 2) YAC-experimental data are very close to EPOS-LHC, QGSJETII-04 and SIBYLL2.1, if we assumed "He-rich" primary composition. Hence, YAC-experimental data is consistent with primary composition with the "Herich" model, that is, YAC-experimental data suggests that the main component responsible for the change of the all-particle spectrum around the "Knee" is composed of nuclei heavier than helium. 3) the "Gaisser's fit" primary model however always gives a higher flux by a factor $1.4-2.0$ than the experimental result and other primary models. It teaches us that there are too many primary 

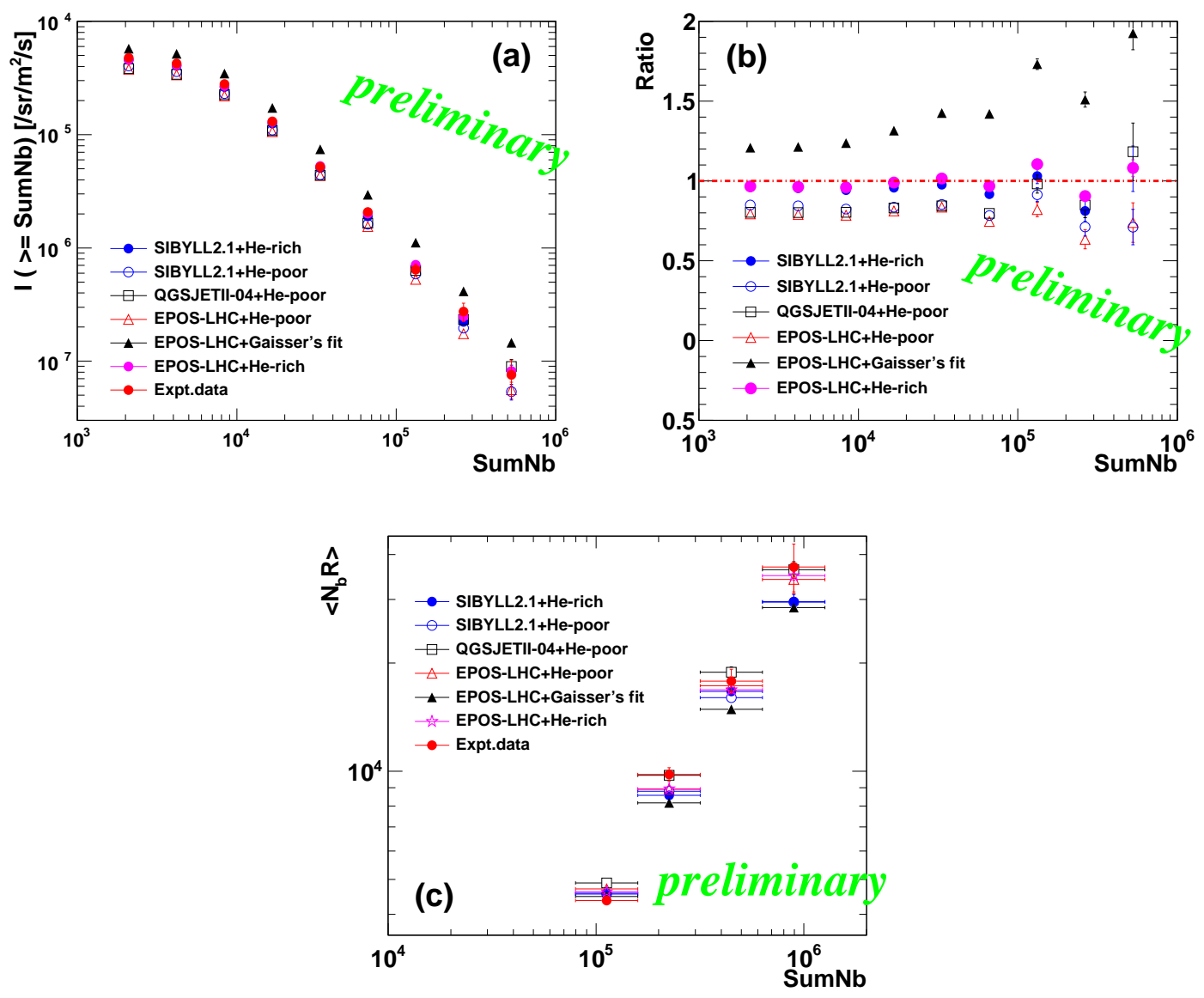

Figure 5: (a) - Integral $\sum N_{b}(\mathrm{SumNb})$ spectra obtained from six MC models and Experimental data, (b) The intensity ratios of $\sum N_{b}$ to that obtained by the Experimental data, the red dash line ( Ratio = 1 ) denoted the Experimental data, (c) - The mean energy-flow lateral spreads $\left\langle N_{b} R\right\rangle$ in the respective energy interval for six MC models and Experimental data.

light components $(\mathrm{P}, \mathrm{He})$ in "Gaisser's fit" model than our experimental data.

The mean lateral spread, $\left\langle N_{b} R\right\rangle$, of AS-cores events is compared with the MC data in Fig.5(c). It is known that, though not strong, it is dependent on the transverse momentum of secondary particles produced in hadronic interactions as well as interaction height. The figure shows some discrepancies among the models: YAC-experimental data are very close to "EPOS-LHC+He-rich". However, the "Gaisser's fit" primary composition model always gives a lower distribution than YAC-I experimental results. This conclusion is the same as the above Fig.5 (a)-(b), that is, there are too many primary light components $(\mathrm{P}, \mathrm{He})$ in "Gaisser's fit" than our expectation.

\section{Summary}

The above mentioned results well show that YAC is very effective in observing the primary components spectra and checking the hadronic interaction models. Based on the "He-poor" primary model, we estimate that the difference of EPOS-LHC, QGSJETII-04, SIBYLL2.1 is within $10 \%$ in our concerned energy region. YAC-experimental data are very close to EPOS-LHC, if we 
assumed "He-rich" primary composition. High energy core events are very sensitive to the light components in CRs and the core parameters are very useful to separate the light components from all the observed events using a ANN technique. Simultaneous observation of core events with YAC and MD array may allow us to obtain the spectrum of each of primary components separately. Actually, the full MC simulation shows that the (YAC-II+Tibet-III +MD) array is powerful enough to obtain the energy spectra of proton, helium, medium nuclei and iron spectra of primary CRs in the range of $50 \mathrm{TeV}-10 \mathrm{PeV}$ with sufficient accuracy. It is estimated that the energy resolution of primary particles of $1 \mathrm{PeV}$ is better than $12 \%$.

\section{Acknowledgments}

The collaborative experiment of the Tibet Air Shower Arrays has been performed under the auspices of the Ministry of Science and Technology of China (No. 2016YFE0125500) and the Ministry of Foreign Affairs of Japan. This work was supported in part by a Grant-in- Aid for Scientific Research on Priority Areas from the Ministry of Education, Culture, Sports, Science and Technology, by Grants-in-Aid for Science Research from the Japan Society for the Promotion of Science in Japan. This work is supported by the Grants from the National Natural Science Foundation of China (Nos. 11533007, 11673041, 11603047 and 11275212), by President's International Fellowship Initiative (PIFI), Chinese Academy of Sciences (No. 2017VMA0030), and by the Key Laboratory of Particle Astrophysics, Institute of High Energy Physics, CAS. This work is supported by the joint research program of the Institute for Cosmic Ray Research (ICRR), The University of Tokyo.

\section{References}

[1] M. Amenomori et al., ApJ 678 (2008) 1165.

[2] J. R. Hörandel, Astropart. Phys. 19 (2003) 193.

[3] M. Shibata, Y. Katayose, J. Huang and D. Chen, ApJ 716 (2010) 1076.

[4] M. Amenomori et al., Phys. Lett. B 632 (2006) 58.

[5] Y. S. Yoon, et al., ApJ 728 (2011) 122.

[6] J. Huang et al., Astropart. Phys. 66 (2015) 18.

[7] L. M. Zhai et al., J. Phys. G: Nucl. Part. Phys. 42 (2015) 045201.

[8] T. Antoni et al., Astropart. Phys. 24 (2005) 1.

[9] Heck D. et al., 1998 Forschungszentrum Karlsruhe Report FZKA 6019

[10] Agostinelli S. et al., Nucl. Instrum. Meth. Phys. Res. A 506 (2003) 250.

[11] M.Amenomori, et al., The Proc. 32nd Int. Cosmic Ray Conf. (Beijing) (HE 1.4,ID: 1217), (2011). 